\title{
Verminoses dos bovinos: percepçáo de pecuaristas em Minas Gerais, Brasil
}

\author{
Cattle helminthiasis: farmers perception in Minas Gerais, Brazil \\ Francisco Eduardo da Fonseca Delgado'; Walter dos Santos Lima²; Arildo Pinto da Cunha ${ }^{1}$; \\ Ana Cristina Passos de Paiva Bello ${ }^{1}$; Luísa Nogueira Domingues ${ }^{1}$; Rebeca Passos Bispos Wanderley; \\ Patrícia Vieira Bossi Leite ${ }^{1}$; Romário Cerqueira Leite ${ }^{3 *}$ \\ ${ }^{1}$ Curso de Pós-Graduação em Ciência Animal, Escola de Veterinária, Universidade Federal de Minas Gerais - UFMG \\ ${ }^{2}$ Instituto de Ciências Biológicas, Departamento de Parasitologia, Universidade Federal de Minas Gerais - UFMG \\ ${ }^{3}$ Departamento de Medicina Veterinária Preventiva, Escola de Veterinária, Universidade Federal de Minas Gerais - UFMG
}

Recebido em 30 de Janeiro de 2009

Aceito em 23 de Março de 2009

\section{Resumo}

O presente trabalho foi realizado com o objetivo de caracterizar o controle dos endoparasitos em rebanhos bovinos do Estado de Minas Gerais. Foram aplicados questionários em 1.304 propriedades rurais, dos quais 1.289 foram validados e utilizados no estudo. As propriedades localizavam-se em 555 municípios inseridos nas 66 microrregióes do Estado de Minas Gerais e foram selecionados por amostragem aleatória. Os entrevistados foram questionados quanto ao perfil sócioeconômico, características de produçáo e de manejo sanitário da populaçáo bovina de cada propriedade, além das atitudes tomadas para o combate dos endoparasitos. Os resultados demonstraram que as diversas práticas de controle das verminoses recomendadas para os rebanhos bovinos são desconhecidas pela maioria dos pecuaristas.

Palavras-chave: Atitudes, pecuaristas, endoparasitos, bovinos.

\begin{abstract}
This study aimed to characterize the cattle endoparasites control in Minas Gerais State. Questionnaires were applied in 1,304 farms and 1,289 were validated and used in this study. The farms were located at 555 municipalities inserted in 66 Minas Gerais micro-regions and were selected by random sampling. The interviewees were asked about socioeconomic profile, production characteristics and cattle sanitary management of each farm, and attitudes to control the endoparasites. Results showed that the parasites control practices recomemended to cattle are unknown to most of the farmers.
\end{abstract}

Keywords: Attitudes, farmers, endoparasites, cattle.

\section{Introdução}

A pecuária bovina é uma atividade de extrema importância econômica para o Brasil. O país possui hoje o maior rebanho comercial do mundo com, aproximadamente, 200 milhóes de animais, dos quais 22,5 milhôes encontram-se em Minas Gerais (IBGE, 2007).

O controle de parasitos em bovinos é um importante fator na produção, uma vez que os parasitos causam grandes perdas econômicas devido à queda de produtividade e transmissão de patógenos, podendo ocasionar morte em alguns animais. As tentativas de combate que, na maioria das vezes, são realizadas

\footnotetext{
*Autor para correspondência: Romário Cerqueira Leite Departamento de Medicina Veterinária Preventiva, Escola de Veterinária, Universidade Federal de Minas Gerais - UFMG,

Av. Antônio Carlos, 6627, São Francisco, CEP 31270-010,

Belo Horizonte - MG, Brasil; e-mail: rcleite@vet.ufmg.br
}

de forma incorreta com uso excessivo e desordenado das bases terapêuticas, também oneram o custo de produção e ainda não alcançam os objetivos de controle. Além disso, implicam na presença de resíduos nos produtos de origem animal. Confirmando-se os altos valores destinados ao controle de parasitos, nos dados do Sindicato Nacional da Indústria de Produtos para a Sáude Animal (SINDAN, 2009) verificou-se que a comercialização de endo e ectoparasiticidas foi responsável por uma expressiva parcela do mercado brasileiro de produtos veterinários, com participação de $34,3 \%$, no ano de 2008.

Fatores ligados às formas de manejo e à sanidade dos animais nos sistemas de produção foram detalhados e correlacionados com co-fatores sociais, culturais, econômicos e políticos por: Breith e Granda (1985) e Prado et al. (1997a, b) no Estado de Minas Gerais; Pereira et al. (1999) no Rio de Janeiro; e Ribeiro et al. (2003) na Bahia. 
Os aspectos epidemilógicos e de controle de helmintos gastrintestinais em bovinos, no Brasil, foram bem estabelecidos por Guimarães (1972) em Minas Gerais; Honer e Bianchin (1993) e Bianchin et al. (1996) no Estado do Mato Grosso do Sul. Tais estudos possibilitaram o surgimento de propostas de tratamento e de manejo que permitiram planejar adequadamente o controle das verminoses nos rebanhos. Porém, na prática, há uma grande distância entre o conhecimento científico e a realidade do controle das parasitoses nos diferentes sistemas de produção (PRADO et al., 1997 a,b; PEREIRA et al., 1999).

Sendo assim, objetivou-se com este trabalho conhecer as práticas dos pecuaristas sobre a verminose em bovinos no Estado de Minas Gerais, com o intuito de criar subsídios para a tomada de açôes em programas de controle parasitário.

\section{Material e Métodos}

O estudo foi realizado em 555 municípios, inseridos nas 66 microrregióes do Estado de Minas Gerais. Foram entrevistadas pessoas físicas que detinham a responsabilidade pelos sistemas produtivos. Foram aplicados questionários, em 1.304 propriedades, dos quais 1.289 foram validados e utilizados no estudo, considerando: duplicidade de formulários, duplicidade de respostas, inegibilidade das informaçôes coletadas e falta de informaçôes sobre a identificação dos pecuaristas e das propriedades. A coleta dos dados foi realizada por técnicos do Instituto Mineiro de Agropecuária (IMA) e por veterinários autônomos, durante o período de maio a julho de 1999.

O questionário utilizado foi ajustado segundo metodologia proposta por Selltiz et al. (1967). As questôes abordaram os seguintes temas: tipo de exploraçấo, área das propriedades, número de bovinos, idade e escolaridade dos entrevistados, critérios de utilização de anti-helmínticos, categorias animais tratadas, tratamento de vacas em lactação, princípio ativo utilizado, conhecimento sobre métodos de controle, grau de importância das parasitoses no rebanho e formas de assistência técnica.

Para a análise das informaçóes foi construído um banco de dados por tabulação e codificação, os quais foram analisados pelo programa SPHINX LEXICA; EURECA V. 5, estabelecendo-se a frequência de cada variável na amostragem levantada.

\section{Resultados e Discussáo}

Pela análise dos resultados, verificou-se que $66,3 \%$ dos rebanhos eram destinados à produção de leite, enquanto $25 \%$ eram sistemas mistos e 8,7\% rebanho de corte. Quanto ao tamanho das propriedades, observou-se o predomínio $(31,2 \%)$ daquelas com extensão territorial acima de 100 hectares (ha) (Figura 1), dado semelhante ao obtido pelo IBGE que foi de 29,03\% (IBGE, 1998). Houve maior frequência de propriedades com rebanhos com 11 a 30 cabeças e mais de 100 cabeças, 26,7 e 26,3\% (Figura 2), respectivamente, o que retrata um cenário de baixa lotaçáo animal por área, baixa produtividade e, consequentemente, subutilização da terra.

No presente estudo, $46,1 \%$ dos produtores ou responsáveis diretos pela produção possuíam acima de 50 anos de idade (Figura 3),

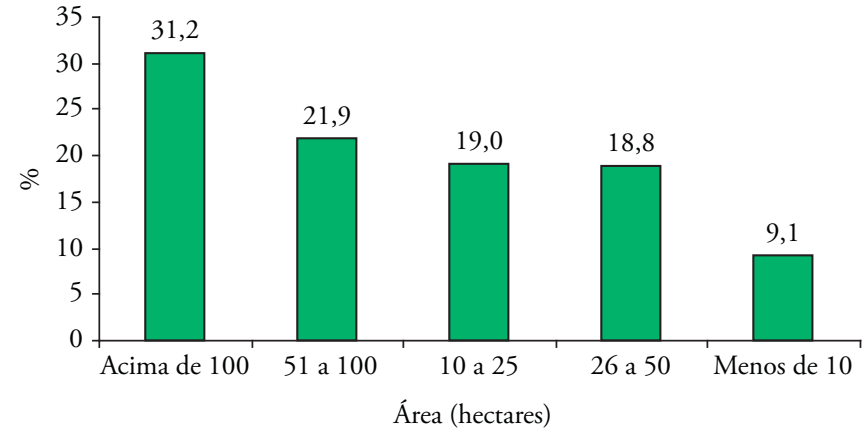

Figura 1. Área utilizada na criação de bovinos, Minas Gerais, 1999.

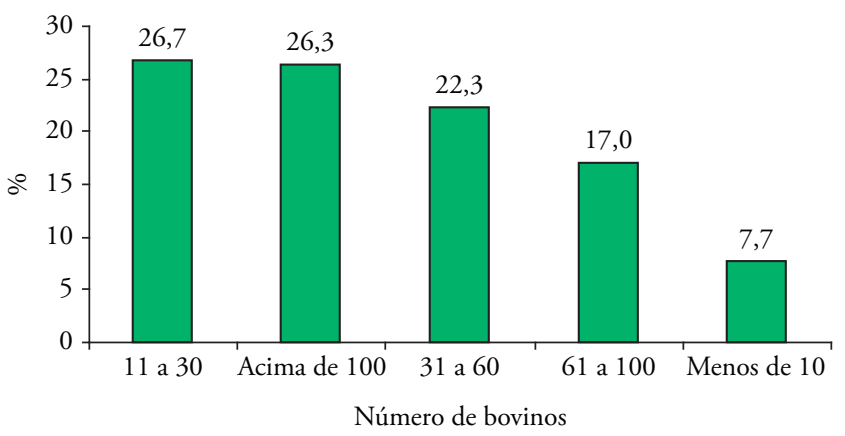

Figura 2. Número de bovinos nos rebanhos, Minas Gerais, 1999.

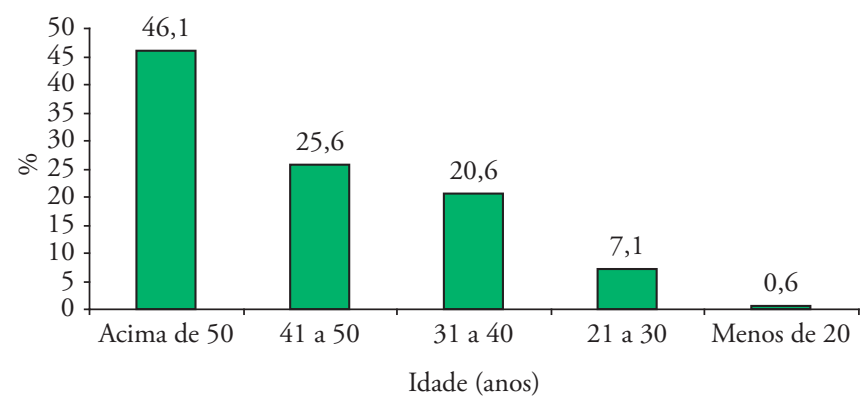

Figura 3. Faixa etária de indivíduos responsáveis por sistemas de criação de bovinos, Minas Gerais, 1999.

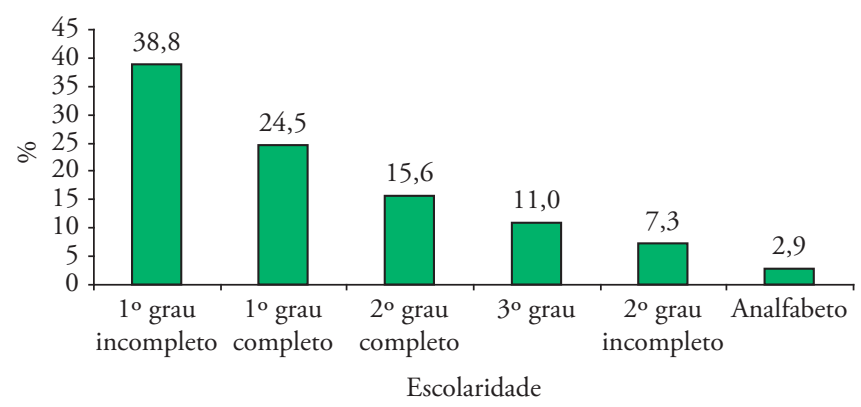

Figura 4. Escolaridade de indivíduos responsáveis por sistemas de criaçáo de bovinos, Minas Gerais, 1999. 
predominantemente com pouca escolaridade (Figura 4). De forma semelhante, Oliveira et al. (2001), em Pedro Leopoldo, Minas Gerais, e Ribeiro et al. (2003), em Ilhéus, Bahia, observaram que a maioria dos produtores entrevistados possuía idade acima de 50 anos. Além disso, dados da Associação Brasileira de Marketing Rural (2000) indicaram que 55\% dos produtores rurais brasileiros apresentaram $1^{\circ}$ grau incompleto. Fatidicamente, essa é a realidade da mão de obra disponível no campo o que, certamente, influencia nas relaçóes sociais e de trabalho e, por consequência nas práticas de manejo. Torna-se, portanto, imprescindível a consideraçáo de tais fatores, somados às particularidades regionais e culturais, nos processos de difusão e transferência de tecnologias.

Quanto ao uso de anti-helmínticos, observou-se a prevalência de medicaçóes mediante quadro clínico (27,3\%) (Figura 5), sendo que $55,9 \%$ tratavam simultaneamente todos os animais do rebanho (Figura 6). Daqueles que dosificavam vacas em lactação, $45 \%$ faziam tratamentos duas vezes ao ano (Figura 7). Charles e Furlong (1996) verificaram que 94,5\% dos produtores de leite dosificavam todas as categorias de animais do rebanho, sendo que $58,2 \%$ dos tratamentos foram realizados sem adoçáo de critérios técnicos e/ou em $40 \%$ das vezes na época inadequada.

Encontrou-se preponderante utilização de avermectina, seguida pelos imidotiazóis e benzimidazólicos (Figura 8). Charles e Furlong (1996), Lima et al. (1985) e Bianchin e Honer (1987) também constataram as avermectinas como os grupos químicos mais utilizados, à frente dos imidotiazóis e benzimidazólicos. A medicação simultânea de todas as categorias animais, juntamente com o uso excessivo e sem critérios técnicos dos anti-helmínticos, principalmente avermectinas, como encontrado neste estudo, podem ser os responsáveis pela acelerada seleção de helmintos resistentes a estes antiparasitários, como já relatado por Rangel et al. (2005), Soutello et al. (2007) e Souza et al. (2008).

Outro achado relevante é de que 69,1\% dos entrevistados náo tinham conhecimento sobre formas de controle de parasitos nos rebanhos (Figura 9). Tais resultados caracterizam o que, de modo geral, ocorre na prática: o manejo sanitário incorreto. $\mathrm{O}$ produtor não utiliza critérios básicos para a tomada das açôes de controle, tais como: o momento, a categoria animal e o uso estratégico do produto anti-helmíntico, pontos fundamentais já pesquisados e divulgados por, Honer e Bianchin (1993) e Bianchin et al. (1996). Embora tais pesquisas existam, as informaçóes não chegam de

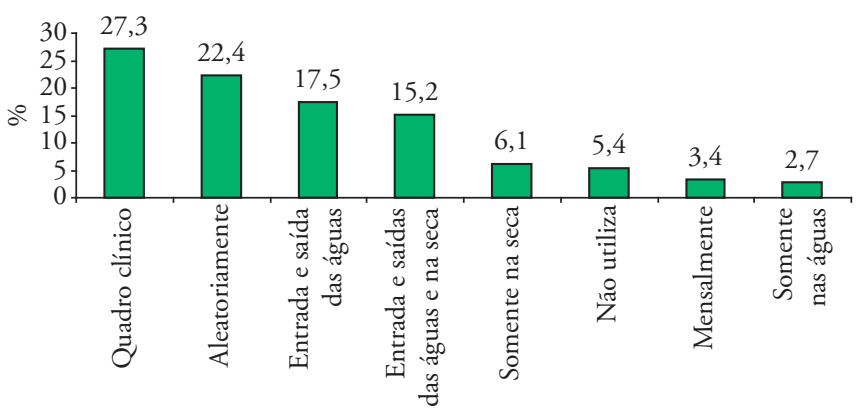

Uso de anti-helmínticos

Figura 5. Critérios para a utilização de anti-helmínticos em bovinos, Minas Gerais, 1999. forma clara e conscistente ao produtor. Segundo Prado (1997a, b), a implantação de tecnologias de controle de doenças não tem levado em consideração a realidade e a percepção socioeconômica e cultural dos aspectos relacionados à sanidade animal.

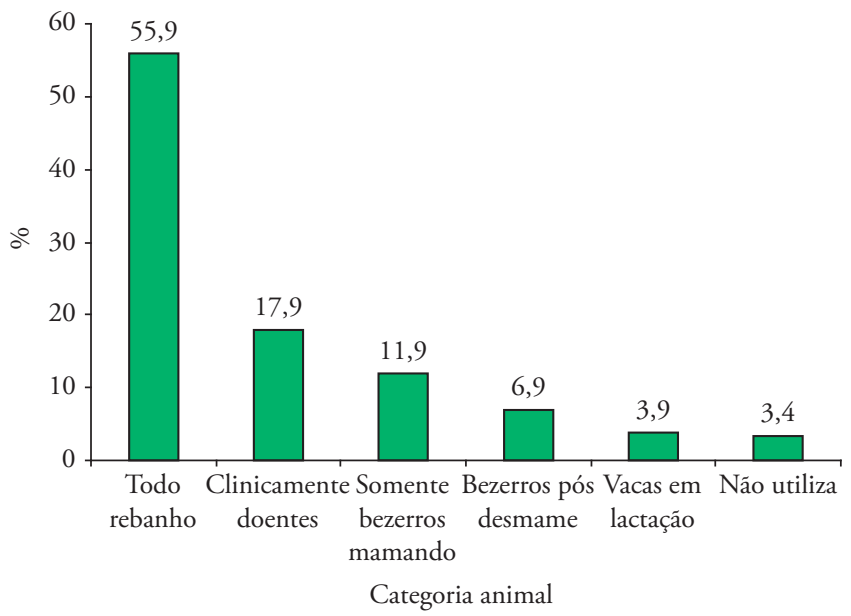

Figura 6. Categoria animal tratada com anti-helmíntico, Minas Gerais, 1999.

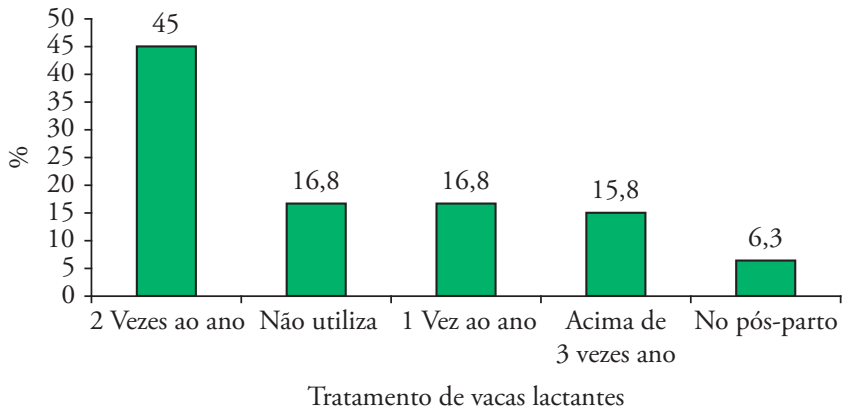

Figura 7. Período e frequência de tratamentos de vacas lactantes, Minas Gerais, 1999.

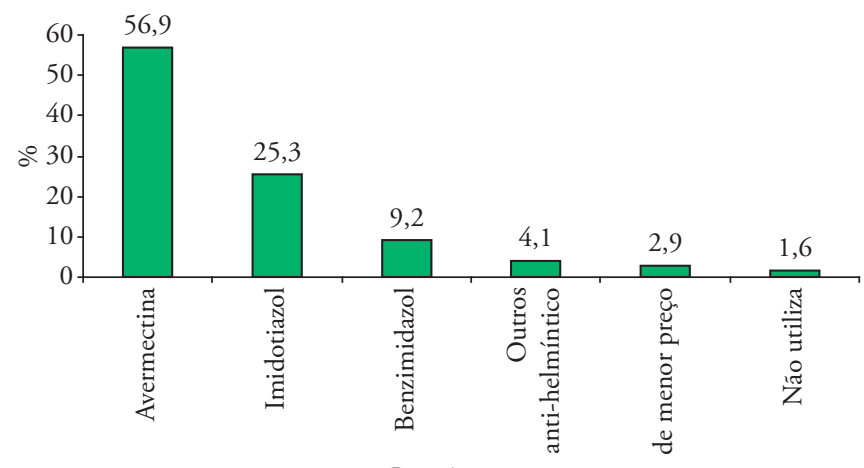

Princípio ativo

Figura 8. Utilização de produtos anti-helmínticos em bovinos, Minas Gerais, 1999. 


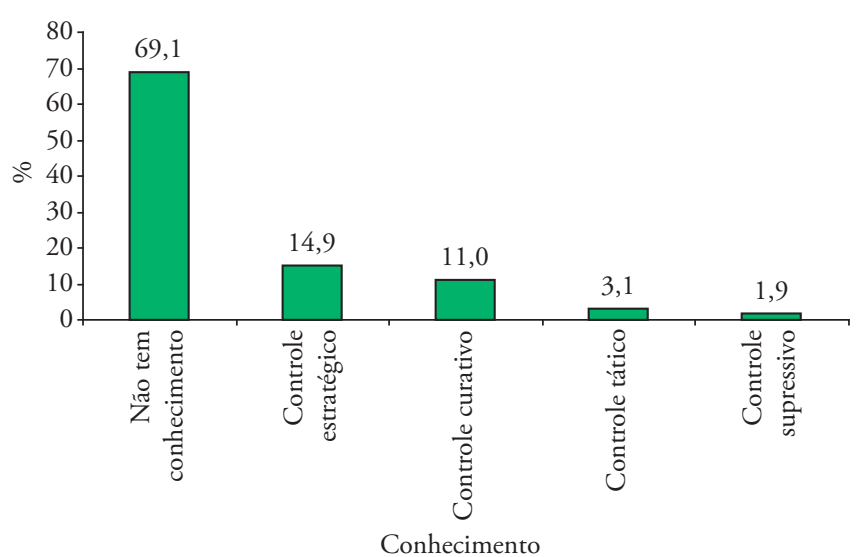

Figura 9. Conhecimento dos entrevistados sobre formas de controle de helmintoses em bovinos, Minas Gerais, 1999.

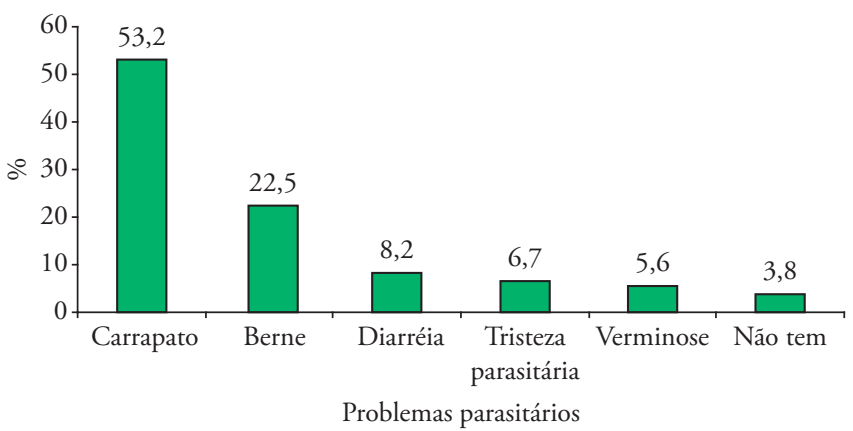

Figura 10. Percepção da importância das parasitoses nos rebanhos bovinos, Minas Gerais, 1999.

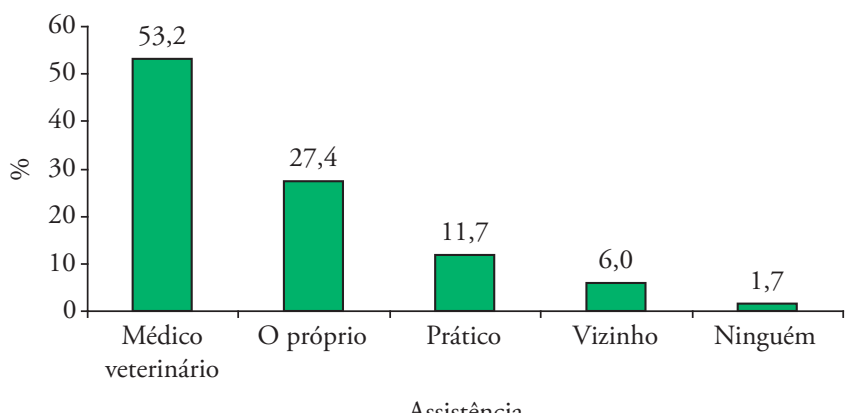

Figura 11. Utilização de assistência técnica para o controle das helmintoses em bovinos, Minas Gerais, 1999.

Os produtores não reconhecem a verminose como um problema parasitário dentro do sistema de produção. Neste estudo, apenas $5,7 \%$ destacaram os endoparasitos como primeira importância, porém expressaram, em 53,2\% das oportunidades, que as infestaçôes por carrapatos constituem a maior preocupação (Figura 10). Souza (2004), em Santa Catarina, relatou que as enfermidades parasitárias são um dos principais fatores de prejuízos na produção de bovinos com destaque para Riphicephalus (Boophilus) microplus, Dermatobia hominis, Haematobia irritans e os helmintos gastrintestinais. Filho (1999) relatou que 60\% dos pecuaristas do agreste do Estado de Pernambuco consideraram o carrapato e a tristeza parasitária como os problemas parasitários mais importantes na bovinocultura, a seguir o berne e a mosca-dos-cifres e, por fim, as helmintoses. Logicamente, os ectoparasitos são melhores e mais frequentemente observados, isto é, são problemas mais visíveis para o produtor em comparação aos parasitos gastrintestinais.

Embora 53,2\% dos entrevistados tenham considerado a preferência pela assistência veterinária, em 39,2\% das vezes admitiram agir por conta própria ou recorrerem ao serviço de "práticos" (Figura 11). Charles e Furlong (1996), Sebastião (1997) e Pereira et al. (1999) demonstraram que a escolha da classe terapêutica e de outras práticas de medicação foram feitas, em primeiro lugar, pelo próprio produtor que, na sequência, buscavam informaçóes junto aos "práticos" e vizinhos.

Em conclusão, as diversas técnicas de controle das parasitoses dos rebanhos bovinos são desconhecidas da maioria dos pecuaristas, os quais não percebem na totalidade os prejuízos provocados pelas endoparasitoses. As estratégias de controle de helmintos em bovinos dependem de um processo de educação sanitária continuada dos agentes envolvidos, a fim de se estabelecer um conhecimento coletivo.

\section{Referências}

Associação Brasileira de Marketing Rural - ABMR. Perfil do produtor rural. São Paulo: ABMR, 2000. Disponível em: <http//www.banet.com. br/propQstalABMR.htm>. Acesso em: Janeiro 2009.

BIANCHIN, I.; HONER, M. R. Helminth parasites of beef cattle in the cerrado region of Brazil. Tropical Animal Health and Production, v. 19 , n. 1, p. 39-45, 1987.

BIANCHIN, I. et al. Epidemiologia dos nematódeos gastrintestinais em bovinos de corte nos cerrados e o controle estratégico no Brasil. Campo Grande: EMBRAPA/CNPGC, 1996. 120 p.

BREITH, J.; GRANDA, E. Os novos rumos da epidemiologia. In: Nunes, E. D. As ciências sociais em saúde na América Latina: tendências e perspectivas. Brasília: OPAS, 1985. p. 241- 253.

CHARLES, T. P.; FURLONG, J. A survey of dairy cattle worm control practices in Southeast Brazil. Veterinary Parasitology, v. 65, n. 1-2, p. 65-73, 1996.

FILHO, J. P. S. Percepçáo e realidade de produtores de leite e médicos veterinários da mesorregiáo do agreste do Estado de Pernambuco sobre o controle da verminose bovina. Rio de Janeiro, 1999. 93 p. Tese (Doutorado em Medicina Veterinária) - Universidade Federal Rural do Rio de Janeiro - UFRRJ.

GUIMARÁES, M. P. Variação estacional de larvas infectantes de nematóides parasitas de bovinos em pastagem de cerrado de Sete Lagoas, MG. Arquivos da Escola de Veterinária da UFMG, v. 24, n. 1, p. $97-113,1972$.

HONER, M. R.; BIANCHIN, I. Programa de controle estratégico da verminose em gado de corte no Brasil. Hora Veterinária, v. 12, n. 71, p. 17-19, 1993. 
Instituto Brasileiro de Geografia e Estatística - IBGE. Censo agropecuário 1995/1996: Distrito Federal, Espírito Santo, Goiás, Mato Grosso, Mato Grosso do Sul, Minas Gerais, Rio de Janeiro, São Paulo. Rio de Janeiro, 1998.

Instituto Brasileiro de Geografia e Estatística - IBGE, 2007. Pesquisa da pecuária municipal: efetivo dos rebanhos. Rio de Janeiro. Disponível em: <http://www.sidra.ibge.gov.br/bda/pecua/default.asp?t=2\&z=t\&o $=22 \& \mathrm{u} 1=1 \& \mathrm{u} 2=1 \& \mathrm{u} 3=1 \& \mathrm{u} 4=1 \& \mathrm{u} 5=1 \& \mathrm{u} 6=1 \& \mathrm{u} 7=1>$. Acesso em: Janeiro 2009.

LIMA, W. S.; GUIMARÁES, M. P.; LEITE, A. C. R. Custo benefício de diferentes dosificações anti-helmínticas em relação ao ganho de peso de bezerros de corte. Pesquisa Agropecuária Brasileira, v. 20, n. 11, p. 1333-1335, 1985.

OLIVEIRA, I. C. S. et al. Situação do controle da verminose em bovinos de leite no Município de Pedro Leopoldo, Minas Gerais. Veterinária Notícias, v. 7, n. 2, p. 103-111, 2001.

PEREIRA, M. J. S. t al. Concepções do processo saúde/doença na produção familiar: uma abordagem epidemiológica qualitativa. Revista da Universidade Rural, v. 21, n. 1-2, p. 117-125, 1999. (Série Ciências da Vida).

PRADO, E. et al. Problemas sanitários do rebanho de leite: percepção dos criadores. Arquivo Brasileiro de Medicina Veterinária e Zootecnia, v. 49, n. 1, p. 19-29, 1997a.

PRADO, E. et al. Forma de produção de gado de leite: visão dos criadores. Arquivo Brasileiro de Medicina Veterinária e Zootecnia, v. 49 , p. $113-121,1997$ b.
RANGEL, V. B. et al. Resistência de Cooperia spp. e Haemonchus spp. às avermectinas em bovinos de corte. Arquivo Brasileiro de Medicina Veterinária e Zootecnia, v. 57, n. 2, p. 186-190, 2005.

RIBEIRO, A. R. P. et al. Aspectos da bovinocultura leiteira no município de Ilhéus - BA. Arquivo Brasileiro de Medicina Veterinária e Zootecnia, v. 55, n. 3, p. 374-376, 2003.

SEBASTIÃO, E. C. O. Avaliaçáo do consumo de fármacos excretáveis pelo leite indicados para gado bovino no âmbito das cooperativas da mesoregiáo metropolitana de Belo Horizonte em 1995. Belo Horizonte, 1997. 113 p. Dissertação (Mestrado em Medicina Veterinária) - Universidade Federal de Minas Gerais - UFMG.

SELLTIZ, C. Métodos de pesquisa nas relaçóes sociais. 2 ed. São Paulo: USP, 1967. 687 p.

Sindicato Nacional da Indústria de Produtos para Saúde Animal SINDAN. Mercado Veterinário. São Paulo, 2008. Disponível em: <http:// www.sindan.org.br/sd/sindan/index.html>. Acesso em: Março 2009.

SOUTEllO, R. G. V.; SENO, M. C. Z.; AMARANTE, A. F. T. Anthelmintic resistance in cattle nematodes in northwestern São Paulo State, Brazil. Veterinary Parasitology, v. 148, n. 3-4, p. 360-364, 2007.

SOUZA, A. P. Controle integrado das principais parasitoses de bovinos. Revista Brasileira de Parasitologia Veterinária, v. 13, n. 1, p. 72-79, 2004.

SOUZA, A. P. et al. Resistência de helmintos gastrintestinais de bovinos a anti-helmínticos no Planalto Catarinense. Ciência Rural, v. 38, n. 5 , p. 1363-1367, 2008. 